\title{
Comprehensive Design Considerations and Noise Modeling of Preamplifier for MEMS Electrometry
}

\author{
J. Jalil, Student Member, IEEE, Y. Ruan, H. Z. Li, and Y. Zhu, Senior Member, IEEE
}

\begin{abstract}
The purpose of the work is to evaluate various intrinsic noise sources that limit the charge resolution of microelectromechanical systems (MEMS) vibrating-reed (VR) electrometers. We demonstrate a method of reducing total equivalent referred-to-input (RTI) noise voltage per square root hertz for an operational amplifier based preamplifier (preamp) circuit topology. The proposed circuit's noise model accurately describes the various noise sources and total RTI noise voltage is experimentally verified. The noise analysis clarifies the path toward a preamp with a noise floor limited by thermal noise due to low valued shunt resistance at its input. Furthermore, during the charge measurement, a variation of sensitivity occurs by changing the isolation (or DC blocking) capacitor of the preamp. This phenomenon also affects the noise performance of the circuit and the overall charge resolution for the electrometer. RTI noise voltage of $290 \mathrm{nV} / \sqrt{ } \mathrm{Hz}$ at $5.2 \mathrm{kHz}$ was measured for $50 \mathrm{M} \Omega$ of shunt resistor and $2 \mathrm{pF}$ of isolation capacitor to implement a prototype of the complete preamp, along with the MEMS device fabricated in siliconon-glass (SOG) process. Finally, we present a charge resolution model of the proposed SOG-MEMS electrometry system. The model's underlying design principles reveal low noise preamps accessible to the circuit designers without tangible circuitries and after-the-fact noise measurement.
\end{abstract}

Index Terms - microelectromechanical systems (MEMS) electrometer, referred-to-input (RTI) noise voltage, preamplifier (preamp), vibrating-reed (VR), charge resolution, sensitivity, silicon-on-glass (SOG).

\section{INTRODUCTION}

A charge sensor or electrometer is extensively used in detecting weak electronic charge signals in various applications, such as detection of bio-analyte, tunneling microscopy, mass spectrometry, ionization current detection in radiation chambers, aerosol science, and quantum computing [1]-[6]. Generally, heterogeneity and substrate differences in sensor manufacturing, excessive inherent noises from sensing elements and electronic circuit as well as external disturbances are just a few of the reasons that limit a micro-charge sensor's output accuracy, which in turn hinders its usage in the above applications. Charge resolution $Q_{n}$ is recognized as one of

J. Jalil, H. Z. Li, and Y. Zhu are with Queensland Micro and Nanotechnology Centre, Griffith University, Nathan, QLD 4111, Australia. (e-mail: y.zhu@griffith.edu.au).

Y. Ruan is with Department of Precision Instruments, Tsinghua University, Beijing, People's Republic of China. (e-mail: ruanyong@mail.tsinghua.edu.cn).

Corresponding authors: Y. Ruan; Y. Zhu.

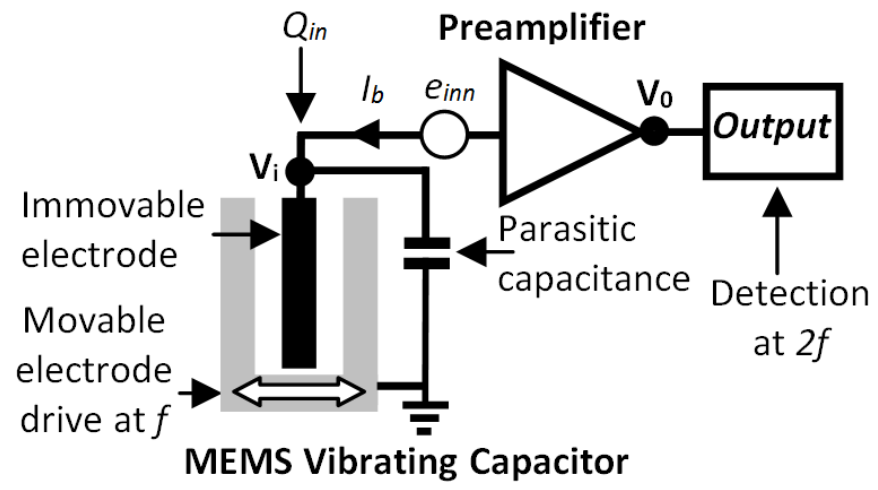

Fig. 1. Schematic diagram of conceptual MEMS vibrating-reed electrometer.

two key parameters to designate the property of electrometry systems as well as sensitivity. Nowadays, a commercial electrometer, for example, Keysight B2987A [7] demonstrates merely $6.3 \mathrm{k}$ electrons of resolution at room temperature and ambient pressure. On the other hand, single electron transistors (SETs) [3], nano-capacitive gate sensing [4] and nanoelectromechanical systems (NEMS) resonator based electrometers [5] detect a fraction of electrons but under stringent conditions of cryogenic temperature and vacuum pressure as well as non-standard fabrication processes. To overcome these issues, microelectromechanical systems (MEMS) based electrometers, such as a resonator type and a vibrating-reed (VR) type are suitable alternatives due to their matured fabrication technologies and operation under room temperature [8]-[16]. However, MEMS resonator type electrometers [8]-[10] require vacuum chambers to achieve a high quality factor to exhibit a better charge resolution. In contrast to resonant electrometers, micromachined VR electrometers [11]-[16] are able to achieve comparably a better charge resolution at room temperature without vacuum conditions.

One of the reasons for poor resolution of commercial electrometers is the use of DC preamplifiers (preamps) with a high magnitude of flicker noise or $1 / f$ noise (near DC levels or low frequencies). To stave off the flicker noise, the MEMS VR electrometer, mainly comprising of a vibrating capacitor with a driving circuit and an AC preamp, modulates a static weak charge signal higher than that of the noise corner frequency within a spectral region of relatively low noise. In these electrometers, a tiny amount of charge (or electrons) is 
deposited on the immovable electrode of the parallel-plates of the vibrating capacitor, as shown in Fig. 1. The charge is then converted to an AC voltage $V_{i}$ due to the movable electrode, which is vibrated by an external force of driving frequency of $f$ in a cyclic manner. Eventually, the AC signal from the output voltage of pramp $V_{0}$ is detected on $2 f$ using signal measurement equipments.

The charge resolution of the electrometer is quantified as [6]

$$
Q_{n}=\frac{e_{i n n}}{S_{e}}
$$

where $S_{e}$ denotes charge-to-rms voltage conversion gain or sensitivity of the MEMS vibrating capacitor, and $e_{i n n}$ denotes total equivalent referred-to-input (RTI) noise voltage (as shown in Fig. 1). $Q_{n}$ represents the minimum input charge required at the electrometer to produce an output signal-to-noise ratio of 1 . In practice, we evaluate $Q_{n}$ by separately measuring referred-to-output (RTO) noise voltage and the charge-to-the output (rms) voltage conversion gain. It is perceived from the above expression, $Q_{n}$ is the function of two attributes: the sensitivity and the total equivalent RTI noise voltage at the frequency of interest. Thus, during charge electrometery at room temperature, both higher the sensitivity of the sensing device and lower noise floor at the input of the preamp stage are of particular importance to achieve higher resolution of the electrometer.

For MEMS VR electrometers, the moving plates of the vibrating capacitor is a mechanical resonator with a micro-meter scale traveling range yielding a relatively small electronic signal during measurement. In this sensing element in $\mathrm{kHz}$ range, the damping coefficient of the thermo-mechanical noise (or Brownian noise) yields a pico-meter range Brownian noise displacement, which corresponds to an infinitesimal electronic signal. Hence, Brownian noise has an insignificant amount of contribution to limit the charge resolution at the output in the vibrating capacitor [6]. On the contrary, the main obstacle in the realization of the high-resolution VR charge sensors is their intrinsic electronic noise sources from the amplifying devices of the preamp, especially at low frequencies below the corner frequency of $1 / f$ noise. At a higher frequency range above the corner frequency of $1 / f$ noise, both Johnson (thermal) and shot noises become dominant. Thus, minimizing the noise of the front-end electronics above the corner frequency of $1 / f$ noise is decisive in obtaining a high-resolution of charge sensors.

Apart from the intrinsic input noises of the amplifying devices, the overall noise of the preamp for MEMS VR charge sensors depends on a number of crucial factors, such as circuit topology, type and process-technology of the semiconductor devices, biasing and gain resistors of the circuit, input capacitance and input resistance of the amplifying device, operating frequency of the vibrating sensor, and sensing element's capacitance and parasitic capacitance due to fabrication technology. To develop a preamp, the circuit designers have control over all of the above factors, except the operating frequency $f$ because it is dictated by the MEMS sensor itself. For low operating frequencies less than $10 \mathrm{kHz}$, the impedance of the input capacitance of the preamp cannot be neglected. Due to the small amplitude of periodic voltage signals from a MEMS
VR sensor, amplification of the input signal must be performed before the output signal can be processed in any way. In addition, the DC input bias or leakage current $I_{b}$ is another paramount issue that needs to be considered (as shown in Fig. 1). The magnitude of the leakage current is required to be as low as possible for the overall efficacy of the readout circuit.

In the last 15 years, researchers have implemented various preamps for MEMS VR electrometer using different approaches. Reihl et al. [11] developed an on-chip CMOS preamp in silicon-on-insulator (SOI)-MEMS process, which integrated buffer circuit, gain amplifier and MEMS switch to reset charge. Lee et al. [12] designed a hybrid MEMS VR electrometer in SOI-MEMS process, where interface circuit was implemented by an off-the-shelf JFET without resetting gate charge arrangement. None of the above circuits were taken any steps to hinder unsolicited input charge from the preamp to the MEMS sensor. Zhu et al. [13] constructed an SOI-MEMS electrometer using an inverting-type operational amplifier (opamp) based preamp with a charge blocking capacitor. To achieve high gain and charge resolution, the feedback capacitors value must be small, for example less than $1 \mathrm{pF}$. However, it is not practical to obtain accurate sub-pF capacitance in PCB implementation due to stray capacitance of the PCBs. Unlike Zhu's capacitor-based preamp, Jaramillo et al. [16] built an SOI-MEMS electrometer using non-inverting resistor-based preamp. The circuit has enough gain to amplify low voltage signal and the utilization of an electronic switch can reset input charge. However, the electrometer is prone to charge drift due to the leakage current in the absence of a charge blocking capacitor. The input leakage current for an opamp based preamp creates two main consequences: shot noise and offset voltage across the external high input impedance. Nowadays, state-of-the-art and miniature opamps are used in designing the preamp circuits in numerous sensing applications because of their continual improvement of noise characteristics [17]-[22]. Moreover, chip manufacturers provide adequate information on opamps which are helpful for circuit designers to characterize those devices accurately. Therefore to comply with the aforementioned design requirements, a commercial off-the-shelf opamp-based preamp is portrayed in this work for a MEMS VR charge sensor.

This paper is focused on the noise characterization and RTI noise reduction methods for opamp based preamplifier, in view of the design of an improved non-inverting topology to overcome the issues of leakage current and charge resetting during measurement. A detailed list of innovations includes: (i) we proposed a comprehensive noise model considered all electronic noise contributors including the preamp in conjunction with capacitive MEMS sensor in silicon-on-glass (SOG) process and ancillary components; (ii) our research findings proved that the blocking capacitor and shunt resistor have strong impact on the RTI noise of the preamp and the charge resolution performance of the electrometer; (iii) the noise analysis and measurement results provided a practical guideline for low noise electrometer interface circuit design; (iv) Trade-offs among opamp selection, blocking capacitor value, shunt resistor value etc. have been discussed to provide an original and in-depth analysis for noise and resolution per- 


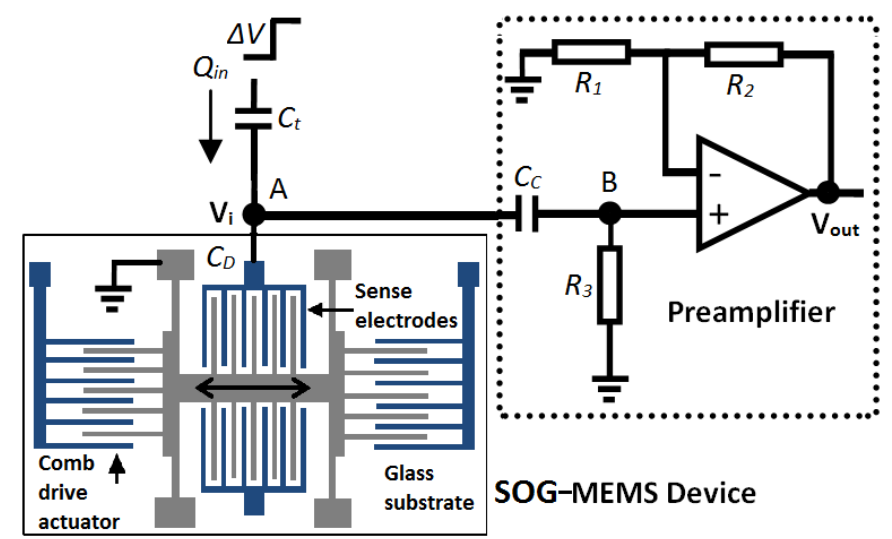

Fig. 2. Schematic of non-inverting voltage-mode preamp for SOGMEMS vibrating-reed electrometer.

formance optimization in the design of MEMS electrometers.

This paper is organized as follows. Section II elucidates the circuit topology of a non-inverting preamp. Noise model of an opamp-based preamp and noise reduction strategies are introduced in Section III. In Section IV, the opamp selection for low noise preamp circuit implementation is illustrated in detail. Section V describes the experimental results and discussion. Lastly, a conclusion is drawn in Section VI.

\section{Circuit Topology of Preamp}

The important issues for opamp-based preamps are: to minimize their noise, to reduce drifting, and to achieve sufficient gain. In addition to the intrinsic noise sources of opamps, charge measurements made with an electrometer are subject to a number of DC error sources from the preamp, including an input leakage current $I_{b}$ and input offset voltage $V_{o s}$. Unavoidable leakage currents are ranging from a few pA to hundreds of $\mathrm{nA}$ for input transistors of the opamp, such as a junction field effect transistor (JFET), metal-oxide semiconductor field effect transistor (MOSFET), and bipolar junction transistor (BJT) [19]. As shown in Fig. 1, if the preamp is directly coupled with the sensor's output, the output of the vibrating capacitor integrates the leakage currents from that preamp, which is seen as a long-term drift in the electrometry. For example, at a fixed temperature, if the input leakage current is $10 \mathrm{pA}$, it causes a change in the charge detection of 10 $\mathrm{pC}$ per second. At extremely low charge levels of $\sim \mathrm{fC}$, even the small valued current might cause a significant error factor measuring the charge. If the input leakage current is known, it is possible to compensate for this error by subtracting the DC charge drift, due to the leakage current from the actual charge detection reading. Nonetheless, precisely determining the leakage current of the entire preamp system might not be an easy task during the measurement. To overcome this issue, a DC blocking or isolation capacitor $C_{C}$ has been introduced in between a sensing device and a preamp, so that the electrons are barred from the opamp's input to the sense electrodes during the charge measurement, as shown in Fig. 2.

In our design, a non-inverting, voltage-feedback opamp with single-pole system has been employed as a voltage-mode preamp, along with a SOG-MEMS charge sensor $C_{D}$, as shown in Fig. 2. Here, a gain resistor $R_{1}$ and a feedback resistor $R_{2}$ are chosen for the voltage gain of the opamp. The input voltage step $(\Delta \mathrm{V})$ can be applied across the off-chip test capacitor $C_{t}$ to set the injected charge $\left(\Delta Q_{i n}=\Delta \mathrm{V} \cdot C_{t}\right)$ at node $\mathrm{A}$. For non-inverting voltage-mode preamps, there are two possibilities to reset the DC leakage currents into the input (node B in Fig. 2) of the opamp during the measurement period. These reset options could be implemented offering a DC (or shunt) path either by switching (non-continuous) mode [16] or by switch-less (continuous) mode [13]. An electronic switch can be used for the switching mode DC path. When the transistorized switch is closed, it couples the non-inverting input of the opamp to the ground. Thereby, it initializes the charge that may have been stored on the plates of the capacitor at the node A. However, this switching shunt circuit requires an external complex control circuitry. Additionally, the switch might result in both parasitic leakage currents and a charge injection, that could be greater than the leakage currents of opamps.

In Fig. 2, a passive shunt resistor of $R_{3}$ has been introduced for direct current path. In the absence of $R_{3}$ at the input terminal of the opamp, the charge due to the intrinsic leakage current from the opamp starts to build on the input capacitor of the opamp, the stray capacitance (due to the wire and the circuit board), and the external non-polarized DC isolation capacitor $C_{C}$. This accumulation of unsolicited charge floats the DC potential at the input of the opamp, and eventually may drive it to the power supply rails making the preamp circuit non-operational. The AC coupling network of $R_{3}-C_{C}$ is placed in between an MEMS sensing device and an opamp as shown in Fig. 2. The fixed non-polarised isolation capacitor $C_{C}$ electrically isolates charge node $\mathrm{A}$ from input node $\mathrm{B}$ of the opamp blocking the leakage current to flow towards node A.

For an opamp as an amlifying device, an initial voltage offset at it's input may induce a corresponding offset charge across the vibrating capacitor without inputting a continuous amount of charge to the node A (as shown in Fig. 2). Hence, a very low voltage offset at the input of opamp is essential to reduce such an initial offset at the output for the betterment of measurement error. However, in our design, both the leakage compensation and the offset correction circuits are circumvented. This is because the electronic components of these circuits contributes more RTI noise at the input of preamp in addition to the preamp circuit itself, and degrades the noise performance of the electrometer.

\section{Noise Model of the Equivalent Circuit}

A circuit noise model is an effective method in order to design a low noise preamp for a certain sensor application. To find the optimum relationships between the elements of our proposed preamp with a MEMS vibrating capacitor, a comprehensive circuit noise model must be introduced. In practice, for a well-designed MEMS VR electrometry system, the noise floor is entirely dominated by the noise characteristics of the preamp. Previously, commercial opamp-based preamps used for SOI-MEMS VR charge sensor described in 
[13] and [16] did not adequately address all of the design criteria to minimize the RTI noise voltage of $e_{i n n}$. Zhu et al. [13] disregarded the equivalent current noise of opamp in their inverting voltage-mode preamp circuit. In this circuit, the equivalent input noise current flows through the sensor's capacitance and the $\mathrm{AC}$ coupling components resulting in an associated noise voltage due to that current noise. Thus, neglecting this noise contributor will cause an error during charge resolution calculation. In their noise model, Jaramillo et al. [16] neither considered the vibrating capacitor with all the parasitic capacitances of both the sensing device and the opamp, nor included the resistance of switching transistor (at off-mode), and subsequently overlooked the noise contribution due to the equivalent input noise current. Hence, it is necessary to identify and quantify all of the component's noise sources of the preamp and account for them while calculating the overall noise of the front-end circuit.

For all band of frequencies, the equivalent input noise voltage $e_{n}$ and current $i_{n}$ of the opamp (originating from their internal components) are the combinations of thermal noise (Johnson noise), shot noise, and flicker noise [19]-[20], [24]-[25]. Unlike flicker noise, both Johnson noise and shot noise are indepenent of frequency. In amplifying devices, Johnson noise occurs from thermal fluctuations in the voltage across a resistor, whereas shot noise stems from the timedependent fluctuations in electrical current due to quantized charges crossing tunnel junctions, p-n junctions and Schottky barrier diodes at random times [25]. It is known that the shot noise of opamps in a $1 \mathrm{~Hz}$ of bandwidth is proportional to the square root of the leakage current, i.e., $i_{n}=\sqrt{2 q I_{b}}$ (where $q$ is the electron charge of $1.6 \times 10^{-19}$ C) [21]. Even though the leakage current in our design has been barred by the isolation capacitor $C_{C}$, it is important to choose an opamp with a low valued leakage current. The reason is while the leakage current of an opamp increases, the shot noise increases as well.

The main noise sources contributed for a monolithic opampbased voltage-mode premp are opamp input noise voltage $e_{n}$, inverting and non-inverting input noise currents $\left(i_{n+}, i_{n-}\right)$ and thermal noises of external resistors $\left(e_{T R 1}, e_{T R 2}, e_{T R 3}\right)$ as shown in Fig. 3. It is worth mentioning that ideal capacitors do not yield thermal noise [24]. The noise contribution of a real capacitor is usually negligible. However, the input noise current $i_{n}$ flowing through it develops the noise voltage. For voltage-feedback opamp, the noise currents in the inverting and noninverting inputs are uncorrelated, i.e. they are two physically independent noise current sources but roughly equal in magnitude. Therefore, matched input transistors in voltagefeedback opamps usually yield the same magnitude for both the noise current sources.

The total referred-to-output (RTO) noise voltage at the frequency of interest of the preamp is computed as the square root of the squared noise contributing terms (also known as the noise power density) to the output node. This computation is summing all the contributing noise power density at the output node by superposition, because all the noise sources are treated as mutually exclusive. The square root is then taken to get back to that of the RTO noise voltage at the frequency of interest. Fig. 3, which represents a noise equivalent circuit

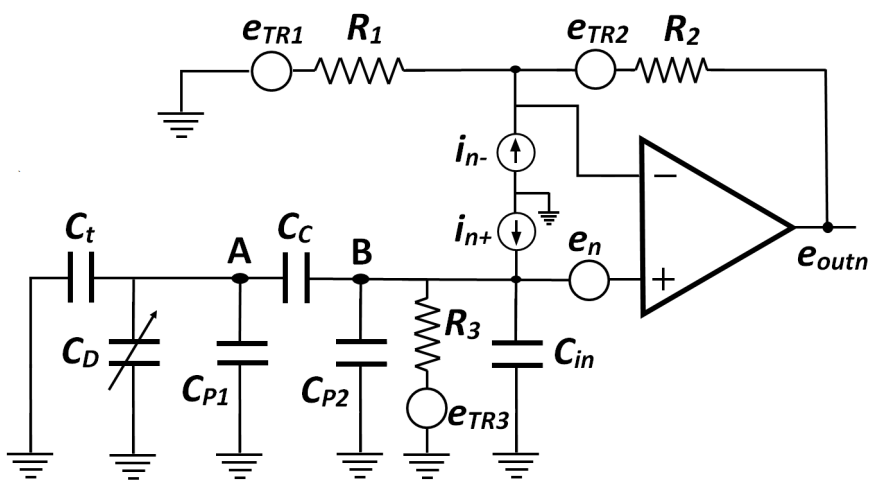

Fig. 3. Noise equivalent circuit of Fig. 2 including all parasitic capacitances.

of Fig. 2, includes a noiseless opamp and associated resistors for designing the preamp, along with MEMS capacitive device $C_{D}$ and its parasitic capacitance of $C_{P 1}$, test capacitor $C_{t}$, as well as input capacitance of the opamp $C_{i n}$ and circuit board layout stray capacitance $C_{P 2}$. Total six noise sources can be identified and calculated individually at the output node $e_{\text {outn }}$ as follows

1. Opamp equivalent input noise voltage spectral density $e_{n}$ and corresponding noise voltage spectral density is

$$
e_{n 01}=e_{n}\left(1+\frac{R_{2}}{R_{1}}\right)
$$

where $\left(1+R_{2} / R_{1}\right)$ is the closed-loop voltage gain of the noninverting opamp. The signal gain and the noise gain for noninverting opamps are equal.

2. Opamp non-inverting equivalent input (shot) noise current spectral density $i_{n+}$ and corresponding noise voltage spectral density is

$$
e_{n 02}=\frac{i_{n+} R_{3}}{\sqrt{1+\omega^{2} C_{\text {total }}^{2} R_{3}^{2}}}\left(1+\frac{R_{2}}{R_{1}}\right)
$$

where $C_{\text {total }}$ is equal to $\left(C_{t}+C_{D}+C_{P 1}\right) \| C_{C}+C_{i n}+C_{P 2}$.

3. Opamp inverting equivalent input (shot) noise current spectral density $i_{n-}$ and corresponding noise voltage spectral density is

$$
e_{n 03}=i_{n-} R_{2}
$$

4. Shunt resistor $R_{3}$ equivalent input thermal noise voltage spectral density $e_{T R 3}$ and corresponding noise voltage spectral density is

$$
e_{n 04}=\sqrt{\frac{4 k T R_{3}}{1+\omega^{2} C_{\text {total }}^{2} R_{3}^{2}}}\left(1+\frac{R_{2}}{R_{1}}\right)
$$

where $k$ is Boltzmann's constant $\left(1.38064852 \times 10^{-23} \mathrm{~J} / \mathrm{K}\right)$, and $T$ is temperature in Kelvin. It is noted that $R_{3}-C_{C}$ network acts as a high-pass filter (HPF) with respect to the modulated charge signal to be measured, while $R_{3}-C_{\text {total }}$ acts as a lowpass filter (LPF) with respect to the thermal noise generated by the shunt resistance $R_{3}$.

5. Gain resistor $R_{1}$ thermal noise voltage spectral density $e_{T R 1}$ and corresponding noise voltage spectral density is

$$
e_{n 05}=\sqrt{4 k T R_{1}} \frac{R_{2}}{R_{1}}
$$


6. Feedback resistor $R_{2}$ thermal noise voltage spectral density $e_{T R 2}$ and corresponding noise voltage spectral density is

$$
e_{n 06}=\sqrt{4 K T R_{2}}
$$

Here, it is considered as $\left|i_{n+}\right|=\left|i_{n-}\right|$. The RTO noise voltage can be expressed (by analyzing Fig. 3) as follows

$$
e_{\text {outn }}=\sqrt{e_{n 01}^{2}+e_{n 02}^{2}+e_{n 03}^{2}+e_{n 04}^{2}+e_{n 05}^{2}+e_{n 06}^{2}}
$$

Voltage transfer function from node A to node B represents as

$$
\frac{V_{B}}{V_{A}}=\sqrt{\frac{\omega^{2} R_{3}^{2} C_{C}^{2}}{1+\omega^{2} R_{3}^{2}\left(C_{C}+C_{i n}+C_{P 2}\right)^{2}}}
$$

The system gain from node $\mathrm{B}$ to node $e_{\text {outn }}$ defined as

$$
A_{v}=\left(1+\frac{R_{2}}{R_{1}}\right) \sqrt{\frac{\omega^{2} R_{3}^{2} C_{C}^{2}}{1+\omega^{2} R_{3}^{2}\left(C_{C}+C_{i n}+C_{P 2}\right)^{2}}}
$$

The total referred-to-input (RTI) noise voltage $\left(e_{i n n}\right)$ per square root $\mathrm{Hz}$ of the preamp circuit is calculated as follows

$$
e_{\text {inn }}=\frac{e_{\text {outn }}}{A_{v}}
$$

Finally, $e_{i n n}$ at node A can be expressed by combining (8), (10), (11), and assuming $\omega^{2} R_{3}^{2}\left(C_{C}+C_{i n}+C_{P 2}\right)^{2} \gg 1$ tending to $R_{3} \gg 5.1 \mathrm{M} \Omega$,

$$
\begin{aligned}
e_{i n n}= & \left(1+\frac{C_{i n}+C_{P 2}}{C_{C}}\right) . \\
& {\left[e_{n}^{2}+\frac{i_{n}^{2}}{\omega^{2} C_{\text {total }}^{2}}+\frac{4 K T}{\omega^{2} R_{3} C_{\text {total }}^{2}}+i_{n}^{2}\left(R_{1} \| R_{2}\right)^{2}\right.} \\
& \left.+4 K T\left(R_{1} \| R_{2}\right)\right]^{\frac{1}{2}}
\end{aligned}
$$

As can be seen from the above equation, all five noise terms depend on the noise factor of $\left(1+\frac{C_{i n}+C_{P 2}}{C_{C}}\right)$. Hence, reducing the value of input and parasitic capacitances of the opamp and increasing the value of isolation capacitance can lessen $e_{i n n}$ considerably. According to the noise term of one, two and four of (12), $e_{i n n}$ can be reduced for a low value of $e_{n}$ and $i_{n}$ of an opamp. To reduce the effect of the second and third noise terms of (12), the operating frequency of the sensor needs to be higher. In this regard, the resonance frequency $f$ of the the sensor's movable electrode has to be a higher range. For femto-ampere of $i_{n}$, the fourth term of (12) can be neglected due to its insignificant magnitude of femto- $\mathrm{V} / \sqrt{ } \mathrm{Hz}$.

At first glance of the preamp circuit as shown in Fig. 3, it might seem that $R_{3}$ needs to be lowered when considering that the higher the resistance value, the higher the thermal noise. But to minimize the noise voltage of the third noise term of (12), the value of $R_{3}$ should be maximized in such a way that the output of the preamp is not being saturated for a fixed value of $C_{C}$. For example, $10 \mathrm{pA}$ of leakage current with 100 $\mathrm{G} \Omega$ of $R_{3}$ yields $1 \mathrm{~V}$ developed across the input of opamp which can potentially cause a complete failure of detecting charge signals from the sensor. Without any input signal, this

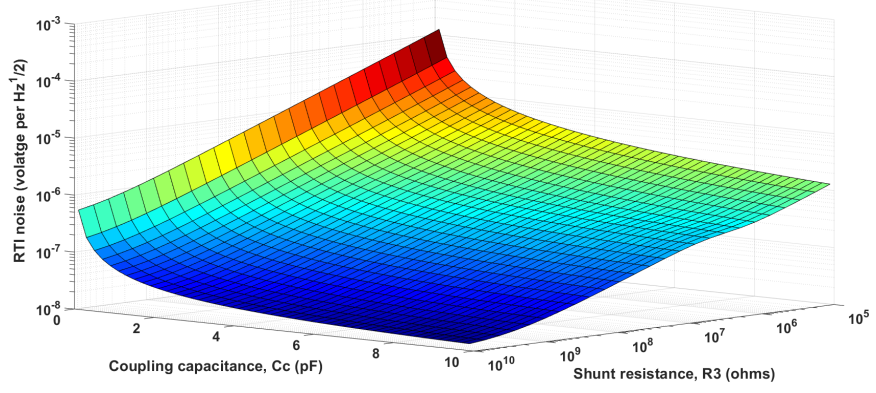

Fig. 4. Calculated RTI noise voltage at $5.2 \mathrm{kHz}$ of (12) as a function of $\boldsymbol{C}_{\boldsymbol{C}}$ and $\boldsymbol{R}_{\mathbf{3}}$.

unwanted DC output error may exhibit a finite output voltage $\left(V_{\text {error }}\right)$. To ensure this output signal is not saturated, closedloop gain of the opamp, leakage current, and offset voltage must be low, and the following design condition needs to be considered

$$
R_{3} \leq \frac{V_{\text {error }}-V_{o s}\left(1+\frac{R_{2}}{R_{1}}\right)}{I_{b}\left(1+\frac{R_{2}}{R_{1}}\right)}
$$

By selecting a very low valued resistances for both gain and feedback resistors, the fourth and fifth noise terms can be made inconsequential for designing a low noise preamp.

Examination of (12) shows that $R_{3}$ and $C_{C}$ are two main parameters that can be manipulated to minimize noise for a given sensor and amplifying device. For the fixed values of $i_{n}$ $=0.6 \times 10^{-15} \mathrm{~A} / \sqrt{ } \mathrm{Hz}$ and $e_{n}=7.5 \times 10^{-9} \mathrm{~V} / \sqrt{ } \mathrm{Hz}$ at $5.2 \mathrm{kHz}$, $k=1.38 \times 10^{-23} \mathrm{~J} / \mathrm{K}, T=298 \mathrm{~K}, C_{P 2}=1 \mathrm{pF}, C_{i n}=2.5 \mathrm{pF}, C_{t}=2$ $\mathrm{pF}, C_{D}=4 \mathrm{pF}, C_{P 1}=1 \mathrm{pF}, R_{1}=49.9 \Omega$, and $R_{2}=680 \Omega$, the total equivalent RTI noise voltage $\left(e_{i n n}\right)$ is calculated using (12) in MATLAB. As shown in Fig. $4, e_{i n n}$ is a function of $R_{3}$ (from $0.1 \mathrm{M} \Omega$ to $10 \mathrm{G} \Omega$ ) and $C_{C}$ (from 0.1 to $3 \mathrm{pF}$ ). By analyzing the graph, a significantly high noise voltage is noticed for lower values of $C_{C}$ and $R_{3}$. On the other hand, for $C_{C}>1.5$ $\mathrm{pF}$ and $R_{3}>10 \mathrm{M} \Omega$, the noise voltage reduces remarkably. The above analysis indicates that considering (12), higher $\mathrm{pF}$ range of $C_{C}$ and higher $\mathrm{G} \Omega$ range of $R_{3}$ are desirable attributes for designing a low noise preamp. According to (12), further noise optimization of preamp can be attained by selecting an opamp having low noise of $e_{n}$ and $i_{n}$.

By analyzing Fig. 2 and Fig. 3 shown previously, the sensitivity for VR electrometer is given as [13]

$$
S_{e}=\frac{C_{D}}{2 \sqrt{2}\left[C_{D}+C_{t}+C_{P 1}+C_{C} \|\left(C_{i n}+C_{P 2}\right)\right]^{2}}\left(\frac{x}{g}\right)^{2}
$$

where $x / g$ is the vibrating plates traveling range ratio of the MEMS device. Finally, using (1) and (14), the charge resolution of our proposed electrometer is represented as

$$
Q_{n}=\frac{e_{i n n}}{8 \sqrt{2} C_{D}}\left[C_{D}+C_{t}+C_{P 1}+C_{C} \|\left(C_{i n}+C_{P 2}\right)\right]^{2}
$$

where the vibrating plates traveling range ratio is half.

\section{OPAMP SELECTION FOR CIRCUIT IMPLEMENTATION}

As mentioned earlier, to fulfil the requirements of preamps for the electrometers, the following input characteristics need 
to be minimized for opamps selection, such as capacitance, DC leakage currents, DC voltage offsets, and noise voltage density and noise current density in the frequency bands of interest. The commercial opamps employing BJTs at the input stage usually demonstrate a low $e_{n}$ but a very high $i_{n}$ [21], [23]. Moreover, the bipolar opamps' open-loop input resistance $R_{i n}$ is significantly smaller than their FET-input counterparts, whereas the input capacitance $C_{i n}$ is more or less similar for both types. In this preamp design, the opamp input resistance value needs to be much higher than $R_{3}$. This is because $R_{i n}$ of an opamp is in parallel with $R_{3}$, and $R_{i n}$ lower than $R_{3}$ limits the overall equivalent input resistance of the preamp. Consequently, regardless a high value of $R_{3}$, the noise performance of the preamp cannot be improved due to the bipolar opamp's low input resistance and high bias current. Therefore, FET-input opamps can be a suitable choice over bipolar opamps as electrometer amplifiers. According to (12), a low $C_{i n}$ is required not only to reduce equivalent input-referred noise but also to help reduce the settling time. However, a low $C_{i n}$ in FETs entails a high $e_{n}$ [25]. Therefore, a design trade-off is involved between the $e_{n}$ and $C_{i n}$ in FETinput opamps.

A handful of commercial ultra-low noise voltage FET-input opamps $\left(e_{n}<10 \mathrm{nV} / \sqrt{ } \mathrm{Hz}\right)$ are found on the market to be worthy as preamps for VR electrometers. For designing low noise preamps, the key properties of ten suitable opamps from leading IC manufacturers are summarized in Table 1. This table reveals performance comparisons among ten different ultra-low noise JFET and MOSFET input opamps in terms of the input-referred noise voltage $e_{i n n}$ and the charge resolution $Q_{n}$ using (12) and (15) at room temperature and $5.2 \mathrm{kHz}$ of frequency. The differences perceived between opamps would be expected as a result of the variation of $e_{n}$, $i_{n}$, and $C_{i n}$. In Table 1 , it is clearly seen that JFET-input opamp of AD8067 from Analog Devices, Inc. [26] exhibits the best charge resolution performance, whereas compared to others, LT1792 exhibits the lowest performance. It is observed that an ultra-low input noise voltage of opamp, for example ADA46251, does not ensure the lowest RTI noise voltage $e_{i n n}$ and thereby a high charge resolution. This is because both $i_{n}$ and $C_{i n}$ also have significant impacts on noise and charge resolution evaluation. Both MAX4475 and MAX40075 MOSFET-input opamps achieve low voltage and current noise at the expense of high $C_{i n}$. Apart from the noise performance, an advantage of using AD8067 opamp in the preamp is its tiny SOT23 package. Even though this opamp strikes a reasoanable balance between $e_{n}, i_{n}, R_{i n}$, and $C_{i n}$ at room temperature, elevated temperatures could increase the leakage currrent $I_{b}$ and might degrade its performance. Finally, this survey shows that commercially available ultra-low noise opamps for MEMS VR electrometry are not yet fully suitable to achieve high charge resolution. The concerns are predominantly achieving low $e_{n}, i_{n}$, and $C_{i n}$ simultaneously for $\mathrm{T} \Omega$ range FET-input opamps.

\section{EXPERIMENTAL RESULTS AND DISCUSSION}

A prototype of the proposed preamp circuit topology was designed using an AD8067 to investigate the sensitivity of
TABLE I

REFERRED-TO-INPUT NOISE VOLTAGE AND CHARGE RESOLUTION COMPARISONS AMONG OFF-THE-SHELF FET-INPUT OPAMPS FOR THE PROPOSED PREAMP CIRCUIT USING (12) AND (15) AT $5.2 \mathrm{KHZ}$ AND ROOM TEMPERATURE.

\begin{tabular}{|c|c|c|c|c|c|c|c|c|}
\hline \multicolumn{6}{|c|}{ Datasheet value } & \multicolumn{3}{|c|}{ Calculated value } \\
\hline No. & $\begin{array}{l}\text { Opamp } \\
\text { model }\end{array}$ & $\begin{array}{l}\text { Input } \\
\text { transistors }\end{array}$ & \begin{tabular}{|l}
$e_{n}$ \\
$(\mathrm{nV} /$ \\
$\sqrt{ } \mathrm{Hz})$
\end{tabular} & $\begin{array}{l}i_{n} \\
(\mathrm{fA} / \\
\sqrt{ } \mathrm{Hz})\end{array}$ & $\begin{array}{l}R_{i n} \\
(\Omega)\end{array}$ & $\begin{array}{l}C_{i n} \\
(\mathrm{pF})\end{array}$ & $\begin{array}{l}e_{\text {inn }} \\
(\mathrm{nV} / \\
\sqrt{ } \mathrm{Hz})\end{array}$ & $\begin{array}{l}Q_{n} \\
\text { (elec./ } \\
\sqrt{ } \mathrm{Hz} \text { ) }\end{array}$ \\
\hline 1. & AD8067 & JFET & 7.5 & 0.6 & $1 \mathrm{~T}$ & 2.5 & 302.8 & 365.1 \\
\hline 2. & ADA46251 & JFET & 3.3 & 4.5 & $1 \mathrm{~T}$ & 8.6 & 298.2 & 393.7 \\
\hline 3. & OPA627 & JFET & 4.85 & 2.5 & $10 \mathrm{~T}$ & 7 & 294.4 & 383.7 \\
\hline 4. & OPA657 & JFET & 4.8 & 1.3 & $10 \mathrm{~T}$ & 4.5 & 296.4 & 374.5 \\
\hline 5. & OPA656 & JFET & 7 & 1.3 & $1 \mathrm{~T}$ & 2.8 & 302 & 367.5 \\
\hline 6. & OPA827 & JFET & 3.9 & 2.2 & $10 \mathrm{~T}$ & 9 & 291.5 & 385.9 \\
\hline 7. & MAX4475 & MOSFET & 4.5 & 0.5 & $1 \mathrm{~T}$ & 10 & 289.2 & 385.1 \\
\hline 8. & LT1792 & JFET & 4.2 & 10 & $100 \mathrm{G}$ & 14 & 327.7 & 443.6 \\
\hline 9. & MAX40075 & MOSFET & 4.2 & 0.5 & $1 \mathrm{~T}$ & 10 & 289 & 384.8 \\
\hline 10. & LTC6244 & MOSFET & 8 & 5 & $1 \mathrm{~T}$ & 3.5 & 310.1 & 484.3 \\
\hline
\end{tabular}

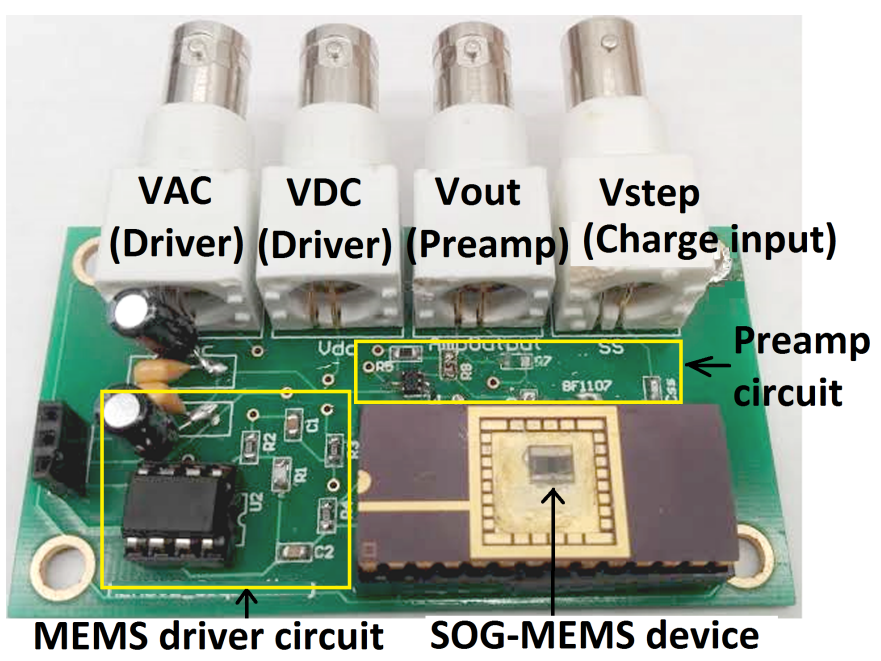

Fig. 5. A photograph of the preamp and the driver circuit on the PCB for SOG-MEMS vibrating-reed electrometer.

a slicon-on-glass (SOG) based MEMS VR charge sensor, as shown in Fig. 5. According to the datasheet of AD8067, $e_{n}$ and $i_{n}$ are $7.5 \mathrm{nV} / \sqrt{ } \mathrm{Hz}$ and $0.6 \mathrm{fA} / \sqrt{ } \mathrm{Hz}$, respectively at 5.2 $\mathrm{kHz}$. During the design of the readout circuit, a feedback resistor and a gain resistor were selected $680 \Omega$ and $49.9 \Omega$, respectively, to achieve an opamp voltage gain of $14.6 \mathrm{~V} / \mathrm{V}$. $R_{3}$ was selected as $50 \mathrm{M} \Omega$, which is much higher than 5.1 $\mathrm{M} \Omega$ to fulfil the condition of (12). The preamp was assembled on a double-sided PCB, where most of the components were surface mount devices. To achieve optimum performance, careful attention was given to a board layout to minimize the stray capacitances. The size of the PCB is $8 \times 4.4 \mathrm{~cm}^{2}$, and it comprises a MEMS driver circuit for actuation, a preamp, and a MEMS IC package holder section. A sample of a SOGMEMS device was wire bonded and packaged in a 28 pin open-cavity ceramic package.

A sinusoidal voltage of $5.5 \mathrm{Vpp}$ along with a DC polarization voltage of $26 \mathrm{~V}$ was applied at $2.6 \mathrm{kHz}$ to drive the SOGMEMS charge device at a temperature of $298 \mathrm{~K}$ and ambient pressure so that the amplitude of the resonator became half of the vibrating capacitor gap. The value of the test capacitance 


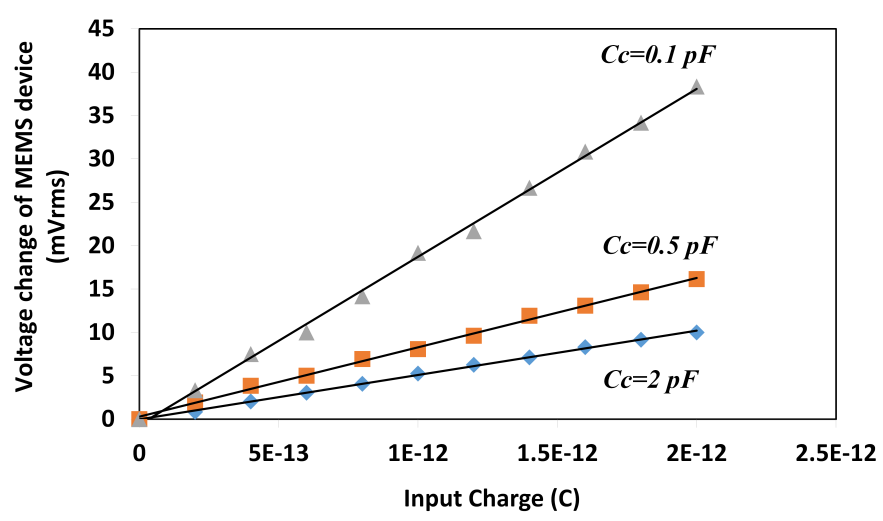

Fig. 6. Sensitivities of SOG-MEMS electrometer for three different values of isolation capacitors.

$C_{t}$ was $2 \mathrm{pF}$. Increasing levels of step voltages were applied to this capacitor using a voltage source. Steps of $0.1 \mathrm{~V}$ to $C_{t}$ were calibrated to the amounts of charge on the fixed terminal of the sensing device, which was the input terminal of the electrometer (See Fig. 2).

The measured output from the preamp was monitored on a mixed signal oscilloscope (MSO-X 3104A, Agilent Technologies) in the fast Fourier transform (FFT) mode. This output (rms) voltage $V_{\text {out }}$ is divided by the system voltage gain of $A_{v}$ to calculate the input voltage $V_{i}$ of the preamp circuit. To keep the opamp and the resistors' parameter reamaining constant, Fig. 6 shows the unamlpified (rms) voltage changes from the SOG-MEMS device with respect to the input charge for three different isolation capacitances of $0.1 \mathrm{pF}, 0.5 \mathrm{pF}$, and $2 \mathrm{pF}$. The sensitivities of $5.06 \times 10^{9} \mathrm{~V} / \mathrm{C}$ and $8 \times 10^{9} \mathrm{~V} / \mathrm{C}$ were achieved with the isolation capacitances of $2 \mathrm{pF}$ and $0.5 \mathrm{pF}$, respectively. On the other hand, the highest sensitivity of $1.9 \times 10^{10} \mathrm{~V} / \mathrm{C}$ was achieved with $C_{C}$ of $0.1 \mathrm{pF}$. It was observed from the readings of Fig. 6 that the charge sensitivity increased by reducing the value of the isolation capacitor.

In the MEMS VR sensor, the injected charge is distributed among the vibrating capacitance of $C_{D}$, the MEMS device parasitic capacitance of $C_{P 1}$, and the equivalent series capacitance of $C_{C} \|\left(C_{P 2}+C_{i n}\right)$ as shown in Fig. 3. Here, the value of $C_{D}$ was $4 \mathrm{pF}$, and this value was higher than the value of the capacitances of $C_{C} \|\left(C_{P 2}+C_{i n}\right)$ and $C_{P 1}$. Thus, most of the input charges accumulate on $C_{D}$, whereas a small amount of charges build on $C_{C}$ and $C_{P 1}$. Regardless of a high PCB parasitic capacitance $C_{P 2}$ and an opamp input capacitance $C_{i n}$, $C_{C}$ in series with these capacitors predominates on $C_{P 2}+C_{i n}$ because of $C_{C}<\left(C_{P 2}+C_{i n}\right)$ based on Fig 3. For the isolation capacitance of $0.5 \mathrm{pF}$, comparably more charge accumulates on to it other than $C_{D}$. Thus, the sensitivity decreases to $8 \times 10^{9}$ $\mathrm{V} / \mathrm{C}$, which is 2.37 times smaller compared to $1.9 \times 10^{10}$ $\mathrm{V} / \mathrm{C}$ (for $C_{C}=0.1 \mathrm{pF}$ ). The sensitivity further decreases by increasing the isolation capacitance to $2 \mathrm{pF}$, as shown in Fig. 6.

It is noteworthy that to evaluate the sensitivity, the shunt resistance $R_{3}$ has no impact on the proposed circuit except $C_{C}$. Although measured reuslts shows low valued $C_{C}$ enhances the sensitivity, it increases the noise contribution according to (12) and affects the resolution performance of the sensor

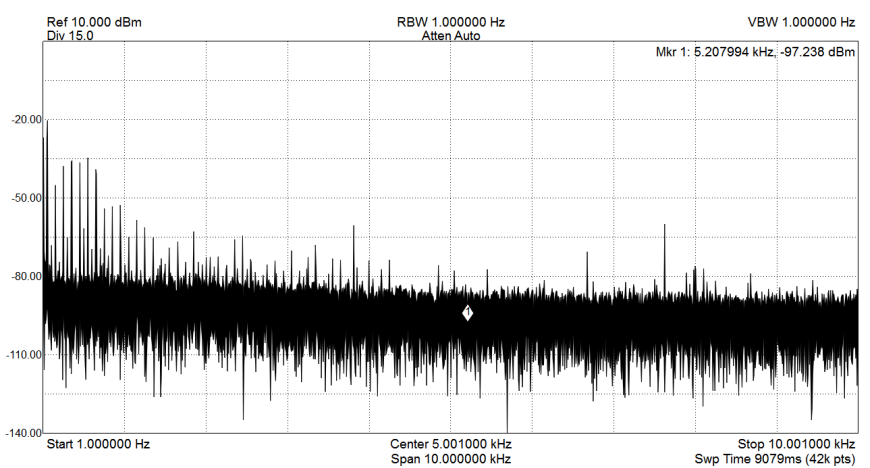

Fig. 7. The measured total RTO noise voltage of the preamp for $\boldsymbol{C}_{C}=2$ $\mathrm{pF}$ and $\boldsymbol{R}_{3}=50 \mathrm{M} \boldsymbol{\Omega}$.

based on (15). To overcome these predicaments, the value of $C_{C}$ has to be increased in such a way that the sensitivity remains high, and the preamp circuit contributes low noise to achieve a high resolution. Apart from $C_{C}$, the test capacitor $C_{t}$ is also a controllable parameter and has an impact on resolution based on equation (15). According to (3) and (5), $C_{t}$ contributes to overall noise. However, in our design, $C_{t}$ (parallel with $C_{D}$ and $C_{P 1}$ ) is in series of $C_{C}$ (as shown in Fig. 3), and $C_{C}<\left(C_{D}+C_{P 1}+C_{t}\right)$. Thus, $C_{C}$ dominates equivalent capacitance of $C_{C} \|\left(C_{D}+C_{P 1}+C_{t}\right)$ in (3), and $C_{t}$ has less effect compared to $C_{C}$ determining RTI noise. On the other hand, according to (15), $C_{t}$ does contribute to the charge resolution. Therefore, $C_{t}$ should be selected as small as possible to enhance the charge resolution performance. During measurements in this work, $C_{t}$ was included to supply a known charge value for sensor's characterization purpose. However, in real applications, such as detection of bio-analyte or aerosol particles, $C_{t}$ will not be there and can be replaced by a conductor or fixed electrode. Hence, this ancillary capacitor's impact is inconsequential in overall charge resolution.

Experimental noise measurement was performed to validate the noise model of the proposed circuit with $C_{C}$ of $2 \mathrm{pF}$ and $R_{3}$ of $50 \mathrm{M} \Omega$. Attained result was cross-checked via simulation. An RTI noise voltage of $317 \mathrm{nV} / \sqrt{ } \mathrm{Hz}$ was achieved for AD8067 by simulating a SPICE compatible circuit simulator, such as TINA-SPICE. An RTO noise voltage of $3.07 \mu \mathrm{V} / \sqrt{ } \mathrm{Hz}$ at $5.2 \mathrm{kHz}$ was measured using a spectrum analyzer (USBSA44B, Signal Hound), as shown in Fig. 7. The output noise measurement was performed in the frequency range from 1 $\mathrm{Hz}$ to $10 \mathrm{kHz}$. The overall gain of the preamp circuit was measured as $10.6 \mathrm{~V} / \mathrm{V}$. Thus, total RTI noise voltage was determined as $290 \mathrm{nV} / \sqrt{ } \mathrm{Hz}$ at $5.2 \mathrm{kHz}$. The noise measurement and noise simulation results of the preamp confirm the theoretical noise model of (12) and gives a direction for further optimization of the circuit. To determine the charge resolution for $C_{C}=2 \mathrm{pF}$, a sensitivity of $5.06 \times 10^{9} \mathrm{~V} / \mathrm{C}$ was considered. Therefore, a charge resolution of $57.31 \mathrm{aC} / \sqrt{ } \mathrm{Hz}$ or 358.2 electrons $/ \sqrt{ } \mathrm{Hz}$ was evaluated.

Both the $R_{3}-C_{C}$ network and the opamp, along with the capacitive MEMS device, have a significant effect on determining the RTI noise voltage of the electrometer to evaluate the overall charge resolution, as shown in the graph of Fig. 8 using (15) and parameters mentioned in section III. A stark 


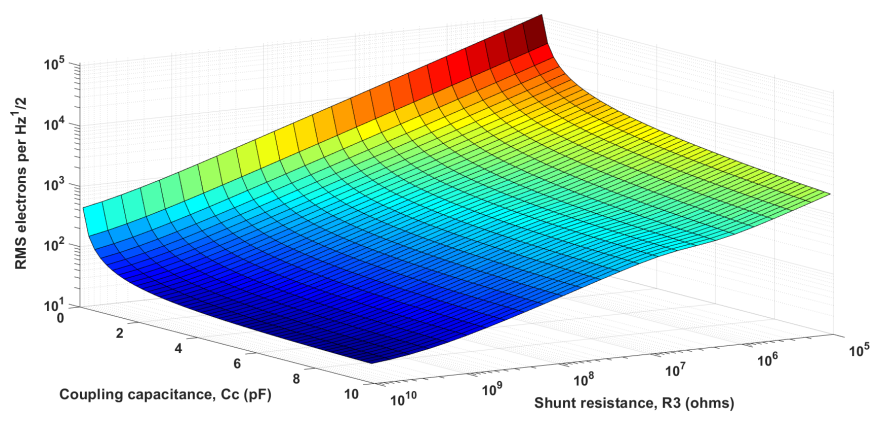

Fig. 8. Calculated RMS electrons of (15) as a function of $\boldsymbol{C}_{\boldsymbol{C}}$ and $\boldsymbol{R}_{3}$.

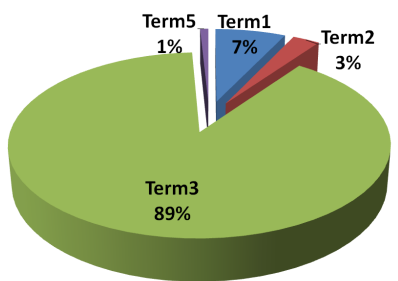

(a)

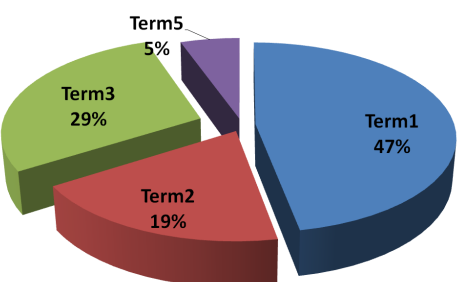

(b)
Fig. 9. Noise contributions of the pramp for (a) $\boldsymbol{R}_{3}=50 \mathrm{M} \boldsymbol{\Omega}$, (b) $\boldsymbol{R}_{\mathbf{3}}=20$ $\mathrm{G} \Omega$.

difference of charge resolution is observed for a high value of $C_{C}$ and $R_{3}$ in comparison with a low value of $C_{C}$ and $R_{3}$. For our designed preamp, Fig. 9 represents the noise contribution from all the noise generators using (12). It was quantified that $89 \%$ of the total noise contributor was the third term of (12) for $R_{3}$ of $50 \mathrm{M} \Omega$, as shown in Fig. 9(a). Thus, lower valued $R_{3}$, which is the dominant noise contributor of $e_{i n n}$, reduces the overall charge resolution based on (15). If $R_{3}$ is increased to $20 \mathrm{G} \Omega, e_{i n n}$ reduces significantly to $28 \mathrm{nV} / \sqrt{ } \mathrm{Hz}$ because of the drastic reduction of the thermal noise of $R_{3}$. In this case, it contributes $29 \%$ of the total noise, as shown in Fig. 9(b). On the other hand, $47 \%$ of the total noise is due to the opamp noise voltage. Hence, for $\mathrm{G} \Omega$ range of shunt resistor, further reduction of RTI noise voltage can be possible by selecting a very low $e_{n}, i_{n}$, and $C_{i n}$ of JFET-input opamps.

\section{CONCLUSIONS}

In this study, we designed a non-inverting voltage-mode preamplifier for charge sensors by analyzing various noise contributors due to the shot noise and the Johnson-Nyquist noise at frequencies above the corner frequency of the flicker noise. With the development of the MEMS based vibratingreed (VR) electrometer, both the sensitivity and a systematic analysis of the noise model for the first-stage of the readout circuit or the preamp have become crucial to quantify the charge resolution. By comparing analytical calculations and experimental measurements, the complete electrometry provides a vivid depiction of its performance rather than just stating the total result. Previously published descriptions of the preamp circuits have neglected a few vital parameters, which are important for evaluating the individual contributions to the charge signal response and the pramp noise. In designing micro-electrometery systems and assessing their sensitivity and resolution by changing electronic circuit parameters, realization of the individual contributions of all relevant parameters are indispensable. The total referred-tothe-input (RTI) noise voltage, in this work, mainly consists of three components: opamp input-referred noise voltage $e_{n}$, opamp non-inverting input-referred noise current $i_{n}$, and noninverting shunt resistor $R_{3}$ thermal noise voltage $e_{T R 3}$. Among them, the thermal noise voltage significantly contributes to high noise due to $\mathrm{M} \Omega$ range of resistance, while the remaining noise components contribute comparably to lower noise. The noise floor detection method was studied for the SOG-MEMS vibrating-reed sensing device with an ultra-low noise AD8067 opamp and an $R_{3}-C_{C}$ network consisting of $50 \mathrm{M} \Omega$ and $2 \mathrm{pF}$. A good correlation was obtained between the theoretical and experimental noise outcomes within the measurement bandwidth of $1 \mathrm{~Hz}$ for $5.2 \mathrm{kHz}$ at room temperature and ambient pressure. Finally, the following decisions and predictions can be stated for the charge resolution model based on theoretical analysis and conducting experiments on the proposed preamp topology for the MEMS VR electrometer:

- Isolation capacitor $C_{C}$ has a considerable effect on both the sensitivity and the RTI noise voltage. To raise the sensitivity, $C_{C}$ must be decreased. On the contrary, lower $C_{C}$ causes high noise voltage leading to a low charge resolution. Thus, $C_{C}$ needs to be increased in favor of the condition $C_{C}<C_{D}$, so that more input charge accumulates on the plates of $C_{D}$ rather than the equivalent series capacitance of $C_{C} \|\left(C_{P 2}+C_{i n}\right)$.

- The value of $R_{3}$ has to be increased to more than $1 \mathrm{G} \Omega$ to achieve high charge resolution provided $1 \mathrm{~T} \Omega$ range of input resistance of the opamp is selected.

- An opamp of ultra-low $e_{n}, i_{n}$, and $C_{i n}$ is a further solution to improve the charge resolution for frequencies above the flicker corner frequency. However, in existing commercial JFET-input opamps, both extremely low $C_{i n}(<1 \mathrm{pF})$ and $e_{n}$ $(<1 \mathrm{nV} / \sqrt{ } \mathrm{Hz})$ are not simultaneously available.

- This method of noise analysis can be used in studying other preamp circuit topologies with any type of vibratingcapacitor based electrometers.

\section{ACKNOWLEDGMENT}

This work was performed in part at the Queensland node of the Australian National Fabrication Facility (ANFF), a company established under the National Collaborative Research Infrastructure Strategy to provide nano and micro-fabrication facilities for Australia's researchers.

\section{References}

[1] H. Paleosky, R. K. Swank, and R. Grenchik, "Design of dynamic condenser electrometers," Rev. Sci. Instrum., vol. 18, no. 5, 298-314, May 1947.

[2] T. R. Ireland, N. Schram, P. Holden, P. Lanc, J. vila, R. Armstrong, Y. Amelin, A. Latimore, D. Corrigan, S. Clement, J. J. Foster, and W. Compston, "Charge-mode electrometer measurements of S-isotopic compositions on SHRIMP-SI," Int. J. Mass. Spectrom., vol. 359, pp. 26-37, Feb. 2014

[3] R. J. Schoelkopf, P. Wahlgren, A. A. Kozhevnikov, P. Delsing, and D. E. Prober, "The radio-frequency single-electron transistor (RF-SET): a fast and ultrasensitive electrometer," Science, vol. 280, no. 5367, pp. 1238-1242, May 1998. 
[4] I. Ahmed, J. A. Haigh, S. Schaal, S. Barraud, Y. Zhu, C. M. Lee, M. Amado, J. W. A. Robinson, A. Rossi, J. J. L. Morton, and M. F. Gonzalez-Zalba, "Radio-frequency capacitive gate-based sensing," Phys. Rev. Applied, vol. 10, no 1, p. 014018, Jul. 2018.

[5] A. N. Cleland and M. L. Roukes, "A nanometer-scale mechanical electrometer," Nature, vol. 392, pp. 160-162, Mar. 1998.

[6] J. Jalil, Y. Zhu, C. Ekanayake, and Y. Ruan, "Sensing of single electrons using micro and nano technologies: a review," Nanotechnology, vol. 28, no. 14, p. 142002, Mar. 2017.

[7] Keysight B2980 Series Femto/Picoammeter Electrometer/High Resistance Meter, Keysight Technologies [Online]. Available: https://literature.cdn.keysight.com/litweb/pdf/B2980-90010.pdf, Accessed on Sep. 28, 2018.

[8] J. E. Y. Lee, B. Bahreyni, and A. A. Seshia, "An axial strain modulated double-ended tuning fork electrometer," Sens. Actuator A-Phys., vol. 148, no. 2, pp. 395-400, Dec. 2008.

[9] D. Chen, J. Zhao, Y. Wang, Z. Xu, and J. Xie, "An electrostatic charge sensor based on micro resonator with sensing scheme of effective stiffness perturbation," J. Micromech. Microeng., vol. 27, no. 6, p. 065002, Apr. 2017.

[10] H. Zhang, W. Yuan, J. Huang, B. Li, and H. Chang, "A high-sensitivity micromechanical electrometer based on mode localization of two degreeof-freedom weakly coupled resonators," J. Microelectromech. Syst., vol. 25, no. 5, pp. 937-946, Oct. 2016.

[11] P. S. Riehl, K. L. Scott, R. S. Muller, R. T. Howe, and J. A. Yasaitis, "Electrostatic charge and field sensors based on micromechanical resonators," J. Microelectromech. Syst., vol. 12, no. 5, pp. 577-589, Oct. 2003.

[12] J. Lee., Y. Zhu, and A. Seshia, "Room temperature electrometry with SUB-10 electron charge resolution," J. Micromech. Microeng., vol. 18, no. 2, p. 025033, Jan. 2008.

[13] Y. Zhu, J. E. Y. Lee, and A. A. Seshia, "A resonant micromachined electrostatic sensor," IEEE Sensors J., vol. 8, no. 9, pp. 1499-1505, Jul. 2008.

[14] J. Jalil, Y. Zhu, T. Dinh, and Y. Ruan, Development of a vibratingreed MEMS charge sensor on silicon-on-glass technology," in Proc. the 5th Int. Confer. Sustainable Design and Manufacturing (KES-SDM18), Gold Coast, Australia, 2018, pp. 126-136.

[15] Y. Zhu, J. Lee, and A. Seshia, "System-level simulation of a micromachined electrometer using a time-domain variable capacitor circuit model," J. Micromech. Microeng., vol. 17, no. 5, pp. 1059-1065, Apr. 2007.

[16] G. Jaramillo, C. Buffa, M. Li, F. J. Brechtel, G. Langfelder, and D. A. Horsley, "MEMS electrometer with femtoampere resolution for aerosol particulate measurements," IEEE Sensors J., vol. 13, no. 8, pp. 29933000, Jun. 2013.

[17] G. Rietveld, "Vibrating reed electrometer with attocoulomb charge resolution and subattoampere current resolution," IEEE Trans. Instrum. Meas., vol. 62, no. 1, pp. 192-198, Jan. 2013.

[18] G. Rietveld and H. E. van den Brom, "Vibrating reed electrometer foraccurate measurement of electrical currents below $10 \mathrm{pA}$," IEEE Trans. Instrum. Meas., vol. 56, no. 2, pp. 559-563, Apr. 2007.

[19] W. M. Leach, Jr., "Fundamentals of low-noise analog circuit design," Proc. IEEE, vol. 82, no. 10, pp. 1515-1538, Oct. 1994.

[20] R. Palls-areny and J. G. Webster, Sensors and Signal Conditioning, New York: Wiley, 2001.

[21] E. Serrano-Finetti and R. Pallas-Areny, "Noise reduction in AC-coupled amplifiers," IEEE Trans. Instrum. Meas., vol. 63, no. 7, pp. 1834-1841, Jul. 2014.

[22] G. Scandurra, G. Giusi, and C. Ciofi, "Multichannel amplifier topologies for high-sensitivity and reduced measurement time in voltage noise measurements," IEEE Trans. Instrum. Meas., vol. 62, no. 5, pp. 1145 1153, May 2013.

[23] F. A. Levinzon, "Measurement of low-frequency noise of modern lownoise junction field effect transistors," IEEE Trans. Instrum. Meas., vol. 54, no. 6, pp. 2427-2432, Dec. 2005.

[24] C. D. Motchenbacher and J. A. Connely, Low Noise Electronic System Design, New York: Wiley, 1993.

[25] S. M. Sze and Kwok K. Ng, Physics of Semiconductor Devices, New Jersey: Wiley, 2007.

[26] Analog Devices, Inc. Datasheet AD8067 [Online]. Available: http://www.analog.com/media/en/technical-documentation/datasheets/AD8067.pdf, Accessed on: Apr. 1, 2017. 